\title{
Juvenile Arthritis Disease Activity Score
}

National Cancer Institute

\section{Source}

National Cancer Institute. Juvenile Arthritis Disease Activity Score. NCI Thesaurus. Code C121354.

A continuous measure of disease activity in juvenile arthritis related to inflammation. This validated measure has established cut points of minimal disease activity, inactive disease and remission. 